E-006

FEASIBILITY AND SAFETY OF MECHANICAL THROMBECTOMY IN STROKE PATIENTS PRESENTING WITH DISTAL ACA OCCLUSIONS - INSIGHTS FROM STAR

${ }^{1} \mathrm{D}$ Pullmann*, ${ }^{1,2}$ E Almallouhi, ${ }^{1,2} \mathrm{~S}$ Al Kasab, ${ }^{3} \mathrm{~A}$ Alawieh, ${ }^{1} \mathrm{R}$ Chalhoub, ${ }^{4} \mathrm{R}$ Starke, ${ }^{5} \mathrm{R}$ De Leacy, ${ }^{6} \mathrm{D}$ Raper, ${ }^{7} \mathrm{~A}$ Rai, ${ }^{8} \mathrm{~T}$ Dumont, ${ }^{9} \mathrm{~S}$ Wolfe, ${ }^{10} \mathrm{P}$ Jabbour, ${ }^{11} \mathrm{C}$ Ogily, ${ }^{12} \mathrm{M}$ Park, ${ }^{13} \mathrm{M}$ Levitt, ${ }^{14} \mathrm{~A}$ Polifka, ${ }^{15} \mathrm{R}$ Crowley, ${ }^{16} \mathrm{~A}$ Arthur, ${ }^{17} \mathrm{~J}$ Osbun, ${ }^{18} \mathrm{R}$ Crosa, ${ }^{19} \mathrm{I}$ Maier, ${ }^{20} \mathrm{~J}$ Kim, ${ }^{21} \mathrm{~W}$ Casagrande, ${ }^{22} \mathrm{~A}$ Shaban, ${ }^{3} \mathrm{~J}$ Grossberg, ${ }^{23} \mathrm{~S}$ Chowdhry, ${ }^{24} \mathrm{M}$ Mokin, ${ }^{25} \mathrm{C}$ Matouk, ${ }^{26}$ I Fragata, ${ }^{27} \mathrm{~S}$ Webb, ${ }^{28} \mathrm{~A}$ Yoo, ${ }^{29} \mathrm{~J}$ Mascitelli, ${ }^{30} \mathrm{M}$ Psychogios, ${ }^{31} \mathrm{M}$ Azab, ${ }^{1} \mathrm{~A}$ Spiotta. ${ }^{1}$ Neurosurgery, Medical University of South Carolina, Charleston, SC; ${ }^{2}$ Neurology, Medical University of South Carolina, Charleston, SC; ${ }^{3}$ Neurosurgery, Emory University, Atlanta, GA; ${ }^{4}$ Neurosurgery, University of Miami Health System, Miami, FL; ${ }^{5}$ Neurosurgery, Mount Sinai Health System, New York, NY; ${ }^{6}$ Neurosurgery, Baylor College of Medicine, Houston, TX; ${ }^{7}$ Neurointerventional Radiology, West Virginia University, Morgantown, WV; ${ }^{8}$ Neurosurgery, University of Arizona, Tucson, AZ; ${ }^{9}$ Neurosurgery, Wake Forest Baptist Health, WinstonSalem, NC; ${ }^{10}$ Neurological Surgery, Thomas Jefferson University, Philadelphia, PA; ${ }^{11}$ Neurosurgery, Beth Israel Deaconess Medical Center, Boston, MA; ${ }^{12}$ Neurosurgery, University of Virginia Health System, Charlottesville, VA; ${ }^{13}$ Neurological Surgery, University of Washington, Seattle, WA; ${ }^{14}$ Neurosurgery, University of Florida, Gainesville, FL; ${ }^{15}$ Neurosurgery, Rush University, Chicago, IL; ${ }^{16}$ Neurosurgeny, University of Tennessee Health Science Center, Memphis, TN; ${ }^{17}$ Neurosurgery, Washington University in St Louis, St. Louis, MO; ${ }^{18}$ Endovascular Neurosurgery, Médica Uruguaya, Montevideo, Uruguay; ${ }^{19}$ Neurology, Universitätsmedizin Göttingen, Göttingen, Germany; ${ }^{20}$ Neurology, Chonnam National University Hospital, Gwangju, Korea, Republic of; ${ }^{21}$ Neurosurgery, Sanatorio Güemes and Hospital de Agudos Juan A Fernandez, Buenos Aires, Argentina; ${ }^{22}$ Neurology, University of lowa, lowa City, IA; ${ }^{23}$ Neurosurgery, NorthShore University HealthSystem, Evanston, IL; ${ }^{24}$ Neurosurgery, University of South Florida, Tampa, FL; ${ }^{25}$ Neurosurgery, Yale School of Medicine, New Haven, CT; ${ }^{26}$ Neuroradiology, Centro Hospitalar Universitário de Lisboa Central, Lisbon, PORTUGAL; ${ }^{27}$ Neuroendovascular, Bon Secours, Greenville, SC; ${ }^{28}$ Neurointerventional Radiology, Texas Stroke Institute, Plano, TX; ${ }^{29}$ Neurosurgery, University of Texas Health Science Center at San Antonio, San Antonio, TX; ${ }^{30}$ Neuroradiology, Universitätsspital Basel, Basel, Switzerland; ${ }^{31}$ Neurosurgery, Damietta Specialized Hospital, Damietta Desert, Egypt

10.1136/neurintsurg-2021-SNIS.102

Background Mechanical thrombectomy (MT) indications for acute stroke treatment have expanded in the last few years to include medium vessel occlusions. However, limited data is available about the safety and efficacy of MT in the distal anterior cerebral artery (ACA) segments (A2/A3). This study aims to assess the feasibility and outcomes of MT in stroke patients presenting with acute $\mathrm{A} 2$ and $\mathrm{A} 3$ occlusions in a large multicenter registry.

Methods This is a retrospective analysis from the Stroke Thrombectomy and Aneurysm registry (STAR) which maintains data from 40 stroke centers in the United States, Europe, Asia, and South America. We included patients who presented with A2/A3 occlusions and were treated with MT using second generation thrombectomy devices between January 1, 2014, and December 31, 2020. Primary outcome of this analysis was the final modified treatment in cerebral infarction (mTICI) score. Other endpoints included the modified Rankin Scale (mRS) score at 90 days and the rate of symptomatic intracranial hemorrhage (sICH).

Results We identified 27 patients who met the inclusion criteria. Median age was 61 (IQR 53-81) years, 15 (55.6\%) were female, and 14 (51.9\%) were white. A2 segment occlusion was seen in $20(74.1 \%)$ patients and A3 segment in 7 (25.9\%) patients. Fourteen (51.9\%) in patients received intravenous tissue plasminogen activator (tPA) prior to MT. Regarding MT technique used, contact aspiration first line (ADAPT) was used in 14 (51.9\%) patients, stent retriever first-line was used in $4(14.8 \%)$ patients, and a combination of both techniques was used in $9(33.3 \%)$. Intraarterial tPA was used in $6(22.2 \%)$ patients. Final mTICI $\geq 2 \mathrm{~B}$ was recorded in $20(74.1 \%)$ patients (13 [48.1\%] had mTICI of 2C or 3). Periprocedural complications were seen in 4 (14.8\%) patients and sICH occurred in 1 (3.7\%) patient. At 90 days, 17 (63\%) patients achieved functional independence (mRS 0-2).

Conclusion In this multicenter study, MT for distal ACA occlusions in the A2/A3 segments seem to be feasible and associated with low complication and symptomatic hemorrhage rates. Future studies are needed to compare the functional outcome of MT versus medical management for stroke patients presenting with $\mathrm{A} 2 / \mathrm{A} 3$ occlusions.

Disclosures D. Pullmann: None. E. Almallouhi: None. S. Al Kasab: None. A. Alawieh: None. R. Chalhoub: None. R. Starke: None. R. De Leacy: None. D. Raper: None. A. Rai: None. T. Dumont: None. S. Wolfe: None. P. Jabbour: None. C. Ogilvy: None. M. Park: None. M. Levitt: None. A. Polifka: None. R. Crowley: None. A. Arthur: None. J. Osbun: None. R. Crosa: None. I. Maier: None. J. Kim: None. W. Casagrande: None. A. Shaban: None. J. Grossberg: None. S. Chowdhry: None. M. Mokin: None. C. Matouk: None. I. Fragata: None. S. Webb: None. A. Yoo: None. J. Mascitelli: None. M. Psychogios: None. M. Azab: None. A. Spiotta: None.

\section{E-007 TIME OF FLIGHT MR ANGIOGRAPHY OF MULTIPLE BRAIN AVMS IN AN HHT PATIENT AT 7 TESLA}

J Massachi* ${ }^{*}$ K Narsinh, M Ballweber, C Jordan, M Vella, T Moore, D Saloner, S Hetts. UCSF, San Francisco, CA

\subsection{6/neurintsurg-2021-SNIS.103}

Introduction Hereditary Hemorrhagic Telangiectasia (HHT) is a genetic condition associated with a high burden of arteriovenous malformations (AVMs) in multiple organs. Brain AVMs (bAVMs) are of particular clinical significance due to the increased risk of morbidity and mortality if ruptured. Patients with HHT can present with multiple bAVMs of varying sizes and lesions of smaller size, while still clinically significant, may not be readily detected on lower field strength MRI scans although they can be visualized on digital subtraction angiography (DSA). To date, several groups have reported initial findings of 7 Tesla (7T) MR Angiography (MRA) in patients with bAVMs and noted that performance of 7T MRA is superior to $1.5 \mathrm{~T}$ for detection of bAVMs, provides higher quality images and inter-rater agreement than 3T MRA, and is comparable to DSA. However, none of these studies evaluated lesions associated with HHT. Here, we describe a pilot investigation of $7 \mathrm{~T}$ Time of Flight MR Angiography (TOF MRA) in a patient who was previously found to have 11 bAVMs on DSA, and compare imaging findings to those identified in earlier scans at lower field strengths.

Materials and Methods Imaging: A volunteer was scanned in a Siemens 7T whole-body human MR system with a Nova Medical 32-channel head coil array. Results from the latest study were compared to reference images collected on GE Signa HDxt whole-body human MR systems at $1.5 \mathrm{~T}$ and $3 \mathrm{~T}$ with a 16-channel head coil array, and Siemens AXIOM-Artis biplane angiography system. Readers: Images were each 